\title{
Lipid levels do not influence the risk of venous thromboembolism
}

\author{
Results of a population-based cohort study
}

\author{
Inge M. van Schouwenburg'; Bakhtawar K. Mahmoodi'1, 2; Ron T. Gansevoort²; Friso L. H. Muntinghe³; Robin P. F. Dullaart4; \\ Hanneke C. Kluin-Nelemans'; Nic J. G. M. Veeger ${ }^{1,5}$; Karina Meijer ${ }^{1}$ \\ 'Division of Haemostasis and Thrombosis, Department of Haematology, University of Groningen, University Medical Centre Groningen, Groningen, The Netherlands; \\ ${ }^{2}$ Department of Nephrology, University of Groningen, University Medical Centre Groningen, Groningen, The Netherlands; ${ }^{3}$ Department of Vascular Medicine, University \\ of Groningen, University Medical Centre Groningen, Groningen, The Netherlands; ${ }^{4}$ Department of Endocrinology, University of Groningen, University Medical Centre Groningen, \\ Groningen, The Netherlands; ${ }^{5}$ Trial Coordination Centre, Department of Epidemiology, University of Groningen, University Medical Centre Groningen, The Netherlands
}

\begin{abstract}
Summary
Studies on the association between lipid profile and venous thromboembolism (VTE) are inconsistent. This could be caused by classical lipoproteins being inferior to apolipoproteins as markers for VTE risk. Therefore, we examined whether apolipoproteins are more strongly related to VTE than lipoproteins. For this analysis we used the PREVEND prospective community based observational cohort study. Levels of apolipoprotein $A 1(A p o A 1)$, apolipoprotein $B(A p o B)$, total cholesterol (TC), high-density lipoprotein (HDL), non-HDL, low-density lipoprotein (LDL), triglycerides (TG), lipoprotein(a), ApoB/ApoA1 and TC/HDL ratio were assessed. Subjects with VTE were identified using databases of the national registries of hospital discharge diagnoses, death certificates, and the regional anticoagulation clinic. Out of 7,627 subjects, 110 developed VTE during a median follow-up of 10.5 years. In both univariate and multivariable analyses no significant associations be-
\end{abstract}

tween apolipoproteins and overall VTE were observed. Of the classical lipoproteins, TC, non-HDL, LDL, TG, and TC/HDL ratio were significantly associated with overall VTE in univariate analysis. Significant associations were no longer present in multivariable analysis. TGL and LDL were significantly associated with unprovoked VTE in univariate analysis. After adjustment for age and sex this significance was lost. No significant associations between (apo-) lipoproteins and provoked VTE were found. We conclude that apolipoproteins are not better in predicting VTE risk than the classical lipoproteins. Our population-based cohort study does not show an association between both apolipoproteins and the classical lipoproteins and VTE risk.

\section{Keywords}

Venous thromboembolism, apolipoprotein, lipoprotein, lipid

\section{Correspondence to:}

Inge M. van Schouwenburg and Karina Meijer

Division of Haemostasis and Thrombosis, Department of Haematology

University of Groningen, University Medical Centre Groningen

Hanzeplein 1, $9713 \mathrm{GZ}$ Groningen, the Netherlands

Tel.: +31 50 3610225, Fax: +31503611790

E-mail: i.m.schouwenburg@umcg.nl; k.meijer@umcg.nl

\section{Financial support:}

The PREVEND Study has been made possible by grants from the Dutch Kidney Foundation. The funding organization is a public institution and had no role in the design and conduct of the study; collection, management, analysis, and interpretation of the data; and preparation, review, or approval of the manuscript.

Received: June 21, 2012

Accepted after minor revision: August 7, 2012

Prepublished online: September 5, 2012

doi:10.1160/TH12-06-0426

Thromb Haemost 2012; 108: 923-929

\section{Introduction}

Growing evidence shows a relationship between arterial and venous thromboembolism (VTE) (1). A possible explanation for this relationship is an overlap in risk factors, such as an abnormal lipid profile. Indeed, the Jupiter trial showed that the use of statins decreases the risk of VTE (2). Two prospective, observational studies also reported a decreased risk of VTE associated with the use of statins $(3,4)$.

Studies on the association between lipid profile and VTE, however, are inconsistent (5-9). These studies mainly focused on classical lipoproteins (i.e. total cholesterol [TC], low-density lipoprotein [LDL], high-density lipoprotein [HDL], triglycerides [TG] and lipoprotein (a) $[\operatorname{lp}(\mathrm{a})])(5,6,10)$. However, in arterial thromboembolism, apolipoproteins and their ratios are possibly stronger predictors for the risk of arterial thromboembolism than the classical lipoproteins (11-13). This may also apply to VTE. Possibly, the inconsistent results on the association between lipid profile and VTE are caused by the use of inferior risk markers which could not optimally detect the effect of an abnormal lipid profile on VTE risk.

For this reason, we examined whether apolipoproteins are more strongly related to VTE than lipoproteins in the large populationbased cohort of the Prevention of REnal and Vascular ENd stage Disease (PREVEND) Study. 


\section{Materials and methods}

\section{Study population and design}

This study was conducted on participants in the PREVEND Study. Details of this study have been published previously (14) and can be found at http://www.prevend.org. In 1997-1998, all inhabitants of the city of Groningen, the Netherlands, aged 28 to 75 years $(\mathrm{n}=85,421)$, were invited to participate in this prospective cohort study, which was designed to investigate the natural course of albuminuria and its relation to renal and cardiovascular disease. A total of 40,856 subjects (47.8\%) responded. Individuals with insulin-dependent diabetes mellitus were excluded from the PREVEND Study since the link between cardiovascular or renal disease and microalbuminuria in this population is well established. As pregnancy may cause temporary microalbuminuria, pregnant women were excluded from the study too. After these exclusions, all subjects with a urinary albumin concentration of $10 \mathrm{mg} / \mathrm{l}$ or greater $(n=7,768)$ were invited and 6,000 agreed to participate. A random control sample of 3,394 subjects with a urinary albumin concentration of less than $10 \mathrm{mg} / \mathrm{l}$ was invited and 2,592 subjects agreed to participate. Taken together, 8,592 subjects took part in the baseline screening and constitute the PREVEND cohort. For the present study, we excluded participants who were non-fasting at the time of first blood sampling $(n=428)$ and those who used lipid-lowering drugs according to either pharmacy or self-report $(\mathrm{n}=537)$. This left us with 7,627 patients for the present analysis.

All participants provided written informed consent. The PREVEND Study was approved by the local medical ethics committee and was conducted in accordance with the guidelines of the Declaration of Helsinki.

\section{Measurements}

Participants visited the outpatient research unit twice for baseline survey. All participants completed a questionnaire on demographics, cardiovascular disease history, smoking habits, alcohol consumption and medication use prior to their first visit. Height and weight were measured, an 8 hour (h)-fasting blood sample was drawn and two 24-h urine samples were collected. Body Mass Index (BMI) was calculated as weight $(\mathrm{kg})$ divided by square of height $\left(\mathrm{m}^{2}\right)$. During the first and second visit blood pressure was measured, in supine position for 10 minutes with an automatic device (Dinamap XL Model 9300, Johnson-Johnson Medical, Tampa, FL, USA). Blood pressure values are given as the mean of the last two recordings of both visits. Hypertension was defined as systolic blood pressure of at least $140 \mathrm{mmHg}$ or diastolic blood pressure of at least $90 \mathrm{mmHg}$, or the pharmacy-confirmed use of antihypertensive drugs (15). Diabetes mellitus was diagnosed by fasting plasma glucose $\geq 7.0 \mathrm{mM}$, according to 1997 American Diabetes Association criteria (16) or pharmacy-confirmed use of oral glucoselowering drugs. Self-reported data on medication use were confirmed using pharmacy-dispensing information from all commu- nity pharmacies in the city of Groningen; drug use was available and complete for $85 \%$ of PREVEND participants. High-sensitivity $\mathrm{C}$-reactive protein (hsCRP) and urinary albumin concentration were determined by nephelometry (Dade Behring Diagnostic, Marburg, Germany). For urinary albumin excretion, the mean of the two samples was used for analysis. Estimated glomerular filtration rate (eGFR) was estimated using the Modification of Diet in Renal Disease study equation, taking into account sex, age, race, and serum creatinine level (17). Subjects were classified as smokers when they reported smoking cigarettes during the previous year.

\section{(Apo-) lipoproteins}

Serum samples for lipid and apolipoprotein measurements were stored at $-80^{\circ} \mathrm{C}$ until analysis. TC was measured by dry chemistry (Eastman Kodak, Rochester, NY, USA), TG were measured enzymatically. HDL was measured using a homogeneous method (direct HDL, AEROSET System; Abbott Laboratories, Abbott Park, IL, USA) (18). LDL was estimated using the Friedewald formula (19). Non-HDL was calculated as the difference between TC and HDL. Serum apolipoprotein B (ApoB), apolipoprotein A1 (ApoA1) and $\operatorname{lp}(\mathrm{a})$ were determined by nephelometry applying commercially available reagents for Dade Behring nephelometer systems (BN II; Dade Behring) (20).

\section{Definition of thrombotic events}

The database of the regional anticoagulation clinic, which monitors the anticoagulant therapy of all inhabitants of the city of Groningen, was used to identify participants of the PREVEND study who developed VTE between study entry and January 1, 2009. As an additional confirmation, the database of the national registry of hospital discharge diagnoses (Prismant, Utrecht, The Netherlands) was searched. Fatal cases were identified with the use of the database of the national registry of death certificates (Central Bureau of Statistics, The Hague/Heerlen, The Netherlands). When available, data on subjects with VTE according to any of the abovementioned databases was confirmed by patients' medical records $(n=89)$. This was carried out by an assessor blinded for the lipid profile of the subject under investigation. Only objectively verified symptomatic thromboembolic events were considered. Deep vein thrombosis had to be confirmed by compression ultrasound, and pulmonary embolism by ventilation/perfusion lung scanning, or by spiral computed tomography. When VTE had occurred at or within three months after exposure to an exogenous risk factor including surgery, trauma, immobilisation for more than seven days, pregnancy, puerperium, the use of oral contraceptives or hormonal replacement therapy, or malignancy, this event was classified as being provoked. VTE was classified as unprovoked when no such risk factor was present. 


\section{Statistical analysis}

To examine the association between (apo-) lipoproteins and their ratios and VTE risk we used a Cox proportional hazard model. The following variables were examined: ApoA1, ApoB, TC, HDL, nonHDL, LDL, TG, lp(a), ApoB/ApoA1 and TC/HDL ratio. As the hazard ratio (HR) did not gradually increase with increasing levels of the (apo-) lipoproteins, these variables could not cot be included as continues variables. Therefore, the risk of VTE was analysed according to the tertiles of the (apo-) lipoproteins. Adjustments were made for age, sex, hypertension, diabetes, urinary albumin excretion, hsCRP and BMI. In these analyses urinary albumin excretion and hsCRP were $\log$ transformed $\left(\log ^{\mathrm{e}}\right)$ to approach a normal distribution. First order interactions between the (apo-) lipoproteins and the variables included in the model were calculated. The HRs are reported with $95 \%$ confidence intervals ( $95 \%$ CI). Observation time started with study entry and ended at time of VTE, end of study (January 2009), death or moving out of the city. Various subanalyses were performed. Firstly, subjects with provoked and unprovoked VTE were analysed separately. Secondly, a sensitivity analysis was performed, in which all analyses were repeated without excluding subjects that were originally excluded based on the use of lipid-lowering medication. Lastly, design-based sensitivity analyses were performed, using survey probability weights (21), to correct for the enrichment of the cohort for subjects with high levels of urinary albumin excretion.

Categorical data are presented as counts and percentages. Continuous variables are presented as medians with interquartile ranges (IQR) when not normally distributed, and as mean with standard deviation (SD) when normally distributed. Statistical difference was tested with the Mann-Whitney U test, Student's t-test and $\mathrm{Chi}^{2}$ test. A two-tailed $\mathrm{p}<0.05$ was considered statistically significant. Statistical analyses were performed using PASW version 18.0 (IBM SPSS, Chicago, IL, USA).

\section{Results}

\section{Study population}

Baseline characteristics are shown in Table 1. Of the 7,627 subjects $49 \%$ were male. Mean age at enrolment was 49 (SD, 13) years. In 1,949 subjects follow-up ended prematurely at time of death $(\mathrm{n}=531,7 \%)$ or moving out of the city $(\mathrm{n}=1,418,19 \%)$. During a median follow-up of 10.5 (IQR, 10.2-10.8) years, 110 subjects developed VTE at a mean age of $61(\mathrm{SD}, 13)$ years. Median time from study inclusion to VTE was 4.9 (1.9-7.6) years. The annual incidence of VTE was 0.15 ( $95 \% \mathrm{CI} ; 0.12-0.18$ ) per 100 person years. In 61 subjects VTE was secondary to an external risk factor, 44 events were unprovoked. In five events the presence or absence of an external risk factor could not be assessed from medical records. Compared to subjects without VTE, subjects who developed VTE had significantly higher TC, non-HDL, LDL, TG and TC/HDL ratio at baseline. They also had significantly higher BMI, hsCRP
Table 1: Clinical characteristics.

\begin{tabular}{|l|l|l|l}
\hline & VTE (n=110) & $\begin{array}{l}\text { No VTE } \\
(\mathbf{n}=7,517)\end{array}$ & P-value \\
\hline ApoA1(g/l) & $1.33(1.18-1.50)$ & $1.36(1.20-1.56)$ & 0.30 \\
\hline ApoB (g/l) & $1.07(0.90-1.25)$ & $1.00(0.82-1.21)$ & 0.05 \\
\hline ApoB/ApoA1 & $0.76(0.64-0.98)$ & $0.74(0.58-0.93)$ & 0.09 \\
\hline Total cholesterol (mM) & $5.83(5.08-6.80)$ & $5.51(4.87-6.30)$ & 0.01 \\
\hline HDL (mM) & $1.22(0.97-1.45)$ & $1.28(1.04-1.57)$ & 0.03 \\
\hline Non-HDL (mM) & $4.64(3.88-5.43)$ & $4.23(3.44-5.06)$ & 0.001 \\
\hline LDL (mM) & $4.25(3.60-5.10)$ & $3.97(3.23-4.74)$ & 0.001 \\
\hline Triglycerides (mM) & $1.29(0.95-1.93)$ & $1.13(0.82-1.65)$ & 0.003 \\
\hline Lipoprotein (a) (g/l) & $0.06(0.03-0.14)$ & $0.05(0.02-0.14)$ & 0.46 \\
\hline TC/HDL & $4.65(3.81-5.93)$ & $4.32(3.32-5.60)$ & 0.003 \\
\hline Age at enrolment (years) & $58(13)$ & $49(13)$ & $<0.001$ \\
\hline Sex (male) & $59(54)$ & $3681(49)$ & 0.34 \\
\hline Hypertension & $46(42)$ & $2172(29)$ & 0.004 \\
\hline Diabetes mellitus & $3(3)$ & $222(3)$ & 1.00 \\
\hline UAE (mg/24 h) & $14.1(8.1-51.9)$ & $9.3(6.3-16.8)$ & $<0.001$ \\
\hline hsCRP (mg/l) & $1.8(1.1-3.9)$ & $1.3(0.5-2.9)$ & $<0.001$ \\
\hline BMI & $29(5)$ & $26(4)$ & $<0.001$ \\
\hline eGFR & $77.1(15.6)$ & $80.9(14.4)$ & 0.01 \\
\hline Smoking & $33(30)$ & $2844(38)$ & 0.11 \\
\hline VTE, venous thrombon & & & \\
\hline
\end{tabular}

VTE, venous thromboembolism; HDL, high-density lipoprotein; LDL, low-density lipoprotein; 'Apo', apolipoprotein; TC, total cholesterol; UAE, urinary albumin excretion; hsCRP, high-sensitivity C-reactive protein; BMI, body mass index; eGFR, estimated glomerular filtration rate. Categorical data are presented as number (\%), continuous variables are presented as medians (IQR) when not normally distributed, and as mean (SD) when normally distributed.

and urinary albumin excretion and lower HDL and eGFR. Furthermore, subjects with VTE were more often hypertensive and were older.

\section{Risk of VTE according to (apo-) lipoproteins}

Inclusion of time dependent covariates in the Cox proportional hazard model showed that the proportional hazards assumption was not violated. Furthermore, there were no relevant significant first order interactions between the (apo-) lipoproteins and the variables included in the models. Table 2 shows the results of the univariate and multivariable analyses of the apolipoproteins in relation to overall VTE. In both univariate and multivariable analyses no significant associations between apolipoproteins and VTE were observed.

Table 3 shows the results of the univariate and multivariable analyses of the classical lipoproteins and their ratios in relation to overall VTE. In the univariate analyses, TC, non-HDL, LDL, TG and TC/HDL ratio were significantly associated with overall VTE. 


\begin{tabular}{|c|c|c|c|c|c|c|}
\hline Variable & $\begin{array}{l}\text { Crude HR } \\
(95 \% \mathrm{CI})\end{array}$ & P-value & $\begin{array}{l}\text { Adjusted } \mathrm{HR}^{\mathrm{b}} \\
(95 \% \mathrm{CI})\end{array}$ & P-value & $\begin{array}{l}\text { Adjusted } \mathrm{HR}^{\mathrm{C}} \\
(95 \% \mathrm{Cl})\end{array}$ & P-value \\
\hline ApoA1 $(n=7243)^{a}$ & & 0.49 & & 0.56 & & 0.85 \\
\hline $2^{\text {nd }}$ tertile & $0.97(0.61-1.52)$ & & $0.93(0.59-1.48)$ & & $1.00(0.62-1.60)$ & \\
\hline $3^{\text {rd }}$ tertile & $0.76(0.46-1.24)$ & & $0.76(0.45-1.27)$ & & $0.87(0.51-1.49)$ & \\
\hline ApoB $(n=7244)$ & & 0.10 & & 0.91 & & 0.77 \\
\hline $2^{\text {nd }}$ tertile & $1.36(0.81-2.29)$ & & $1.01(0.59-1.71)$ & & $0.85(0.50-1.44)$ & \\
\hline $3^{\text {rd }}$ tertile & $1.71(1.04-2.80)$ & & $1.10(0.66-1.83)$ & & $0.83(0.49-1.41)$ & \\
\hline ApoB/ApoA1 $(n=7243)$ & & 0.17 & & 0.65 & & 0.29 \\
\hline $2^{\text {nd }}$ tertile & $1.59(0.96-2.61)$ & & $1.22(0.73-2.03)$ & & $1.02(0.61-1.71)$ & \\
\hline $3^{\text {rd }}$ tertile & $1.48(0.89-2.45)$ & & $1.01(0.59-1.73)$ & & $0.72(0.41-1.25)$ & \\
\hline
\end{tabular}

Table 2: Risk of venous thromboembolism related to apolipoproteins and their ratios.

Apo, apolipoprotein; $\mathrm{Cl}$, confidence interval. ${ }^{\mathrm{a}}$ number of subjects included in univariate analysis. ${ }^{\mathrm{b}}$ Reference group are those within the first tertile. Adjustments are made for age and sex. ${ }^{C}$ Reference group are those within the first tertile. Adjustments are made for age and sex, hypertension, diabetes, albuminuria, C-reactive protein, Body Mass index, estimated glomerular filtration rate and smoking. HR, hazard ratio;

\begin{tabular}{|c|c|c|c|c|c|c|}
\hline Variable & $\begin{array}{l}\text { Crude HR } \\
(95 \% \mathrm{Cl})\end{array}$ & P-value & $\begin{array}{l}\text { Adjusted } \mathrm{HR}^{\mathrm{b}} \\
(95 \% \mathrm{CI})\end{array}$ & P-value & $\begin{array}{l}\text { Adjusted } \mathrm{HR}^{\mathrm{c}} \\
(95 \% \mathrm{CI})\end{array}$ & P-value \\
\hline $\begin{array}{l}\text { Total cholesterol } \\
(\mathrm{n}=7577)^{\mathrm{a}}\end{array}$ & & $0.045^{*}$ & & 0.61 & & 0.78 \\
\hline $2^{\text {nd }}$ tertile & $1.18(0.71-1.95)$ & & $0.87(0.52-1.45)$ & & $0.83(0.49-1.41)$ & \\
\hline $3^{\text {rd }}$ tertile & $1.73(1.09-2.74)$ & & $1.09(0.68-1.77)$ & & $0.86(0.52-1.43)$ & \\
\hline HDL $(n=7445)$ & & 0.09 & & 0.24 & & 0.82 \\
\hline $2^{\text {nd }}$ tertile & $0.91(0.59-1.40)$ & & $0.96(0.62-1.49)$ & & $1.17(0.72-1.88)$ & \\
\hline $3^{\text {rd }}$ tertile & $0.59(0.36-0.96)$ & & $0.66(0.39-1.11)$ & & $1.08(0.61-1.91)$ & \\
\hline Non-HDL ( $n=7412)$ & & $0.02^{*}$ & & 0.61 & & 0.80 \\
\hline $2^{\text {nd }}$ tertile & $1.39(0.83-2.32)$ & & $1.00(0.59-1.69)$ & & $0.84(0.49-1.45)$ & \\
\hline $3^{\text {rd }}$ tertile & $1.97(1.22-3.18)$ & & $1.21(0.73-2.01)$ & & $0.86(0.50-1.46)$ & \\
\hline LDL $(n=7412)$ & & $0.003^{*}$ & & 0.27 & & 0.93 \\
\hline $2^{\text {nd }}$ tertile & $1.47(0.86-2.49)$ & & $1.07(0.62-1.84)$ & & $0.93(0.53-1.63)$ & \\
\hline $3^{\text {rd }}$ tertile & $2.28(1.40-3.72)$ & & $1.43(0.85-2.40)$ & & $1.02(0.59-1.75)$ & \\
\hline Triglycerides $(n=7446)$ & & $0.01^{*}$ & & 0.10 & & 0.21 \\
\hline $2^{\text {nd }}$ tertile & $2.11(1.26-3.55)$ & & $1.76(1.04-2.97)$ & & $1.36(0.79-2.32)$ & \\
\hline $3^{\text {rd }}$ tertile & $2.16(1.29-3.63)$ & & $1.62(0.96-2.75)$ & & $0.92(0.52-1.63)$ & \\
\hline Lipoprotein (a) (n=7243) & & 0.72 & & 0.83 & & 0.94 \\
\hline $2^{\text {nd }}$ tertile & $1.13(0.69-1.85)$ & & $1.10(0.67-1.80)$ & & $1.00(0.60-1.65)$ & \\
\hline $3^{\text {rd }}$ tertile & $1.21(0.76-1.93)$ & & $1.15(0.72-1.84)$ & & $1.08(0.67-1.73)$ & \\
\hline TC/HDL (n=7412) & & $0.03^{*}$ & & 0.26 & & 0.31 \\
\hline $2^{\text {nd }}$ tertile & $1.94(1.18-3.21)$ & & $1.53(0.92-2.57)$ & & $1.06(0.62-1.81)$ & \\
\hline $3^{\text {rd }}$ tertile & $1.82(1.09-3.03)$ & & $1.29(0.75-2.21)$ & & $0.74(0.42-1.32)$ & \\
\hline \multicolumn{7}{|c|}{$\begin{array}{l}\mathrm{HDL} \text {, high-density lipoprotein; } \mathrm{LDL} \text {, low-density lipoprotein; } \mathrm{TC} \text {, total cholesterol; HR, hazard ratio; } \mathrm{Cl} \text {, confidence interval. } \\
\text { * Statistical significance at two-tailed } \mathrm{p} \leq 0.05 .{ }^{a} \text { number of subjects included in univariate analysis. }{ }^{\mathrm{b}} \text { Reference group are those } \\
\text { within the first tertile. Adjustments are made for age and sex. }{ }^{c} \text { Reference group are those within the first tertile. Adjustments are } \\
\text { made for age and sex, hypertension, diabetes, albuminuria, C-reactive protein, Body Mass index, estimated glomerular filtration } \\
\text { rate and smoking. }\end{array}$} \\
\hline
\end{tabular}

Table 3: Risk of venous thromboembolism related to classical lipoproteins and their ratios. 
However, after adjustment for age and sex, a significant association was no longer present in all these variables. This was also true after additional adjustment for hypertension, diabetes, urinary albumin excretion, hsCRP and BMI.

When analyses were confined to subjects with unprovoked VTE we observed a significant association with TGL and LDL in the univariate analysis. After adjustment for age and sex, no significant associations between unprovoked VTE and any of the (apo-) lipoproteins or their ratios remained. When confined to subjects with provoked VTE no significant associations between any of the variables and VTE was found in both univariate and multivariable analyses.

When a sensitivity analysis was performed in which subjects on lipid-lowering drugs were not excluded from analyses, similar results were found. In the univariate analyses non-HDL, LDL, TG and TC/HDL ratio were related to VTE risk. After adjustment for age and sex, no significant relationship between any of the variables and VTE was found.

When the analyses were adjusted for the enrichment of the cohort with participants with higher urinary albumin levels, using survey probability weights (21), similar results were obtained. In the multivariable analyses no significant relationship between any of the variables and VTE was found.

\section{Discussion}

The present population-based cohort study did not show a stronger association with VTE for apolipoproteins than for classical lipoproteins. Both apo- and lipoproteins showed no association with VTE, neither did their ratios. When analysed separately for subjects with unprovoked or provoked VTE similar results were found.

An association between lipid levels and VTE risk was hypothesised based on the established relationship between cardiovascular disease and VTE (1, 22-24). Possibly, the relationship between the two diseases can be explained by an overlap in risk factors, such as an abnormal lipid profile. The lipid profile is largely influenced by food intake (25), and dietary patterns are strongly related to the risk of cardiovascular disease (26-28). Diet patterns might also be related to VTE risk (29). However, the present study showed that, like many cardiovascular risk factors $(5,6)$, an altered lipid profile does not influence VTE risk. This might explain why the relationship between healthy diets and VTE risk was not always corroborated $(28,30)$. As abnormal lipid levels do not seem to influence VTE risk, we can conclude that the association between cardiovascular disease and VTE is not explained by an abnormal lipid profile.

Previously, a case-control study of 49 male VTE patients and matched controls, aged $<55$ years, showed that VTE risk was elevated in subjects with an elevated ApoB/ApoA1 ratio. When analysed separately low ApoA1 was associated with increased VTE risk while high ApoB level was not (8). On the contrary, Everett et al. reported that high levels of both ApoA1 and ApoB100 were associ- ated with an increased risk of unprovoked VTE in women on hormone therapy (7). In contrast to abovementioned studies, our study was performed in a population-based setting. This might explain the differences in outcome. Everett et al. also did not show a relationship between apolipoproteins and VTE in women who were not on hormone therapy (7). Our findings are in accordance with the findings of a large population based cohort study in which no association between ApoA1 and VTE risk could be demonstrated (31).

Although not confirmed in a meta-analysis (13), two large casecontrol studies suggest that apolipoproteins are better than the classical lipid biomarkers in predicting the risk for arterial cardiovascular disease $(11,12)$. The pathophysiology behind this finding is not fully understood, but is partly explained by the fact that apolipoproteins better reflect an individual's atherogenic potential (32). Atherogeneity will most likely not influence VTE risk, because atherosclerosis does not take place in the venous system. Still, statins reduce the risk of VTE (2-4). Possibly, lipid biomarkers have other characteristics that could influence venous haemostasis. Indeed, it has been demonstrated that an elevated LDL level accelerates activation of prothrombin, factor $\mathrm{X}$ and factor VII, while HDL enhances the protein $C$ anticoagulant pathway and reduces thrombin generation (33). Furthermore, a high level of TC enhances platelet thrombus formation (34). However, the present study did not show an association between the lipid biomarkers and VTE risk. This suggests that the prothrombotic effects of an abnormal lipid profile might be too mild to actually influence VTE risk or that these effects are counteracted by other mechanisms. The reported decreased risk of VTE due to statins (2-4) is probably not caused by lowering lipid levels itself, but more likely through other properties of this medication. This theory is supported by the finding that of all lipid-lowering medication, only statins reduce the risk of VTE (35). Statins have several other mechanisms that could reduce VTE risk. For example it induces Kruppel-Like Factor 2 expression, which in turn promotes thrombomodulin expression on endothelial cells, thereby enhancing the activity of the protein $\mathrm{C}$ anticoagulant pathway (36). Furthermore, statins diminish levels of inflammatory markers $(37,38)$ and they reduce tissue factor expression and thrombin generation (39).

Our study has both strengths and limitations. Strength of the study is that all important lipid biomarkers and the ratios known to be related to arterial cardiovascular disease have been examined. Including all important lipid biomarkers and ratios strengthens the finding that an altered lipid profile does not influence VTE risk. Other strengths of our study are the large population-based cohort and the accurate information on the use of lipid-lowering medicine, which made it possible to exclude these subjects in our analysis.

A limitation of our study is that incidence of VTE may have been underestimated as cases were identified retrospectively. Nonetheless, the assessor who evaluated patient charts was blinded to lipid profile and there is no reason to assume an unequal distribution of missed events between different levels of (apo-) lipoproteins. Second, data regarding anticoagulant therapy were only available for the subjects who developed VTE during follow-up. 


\section{What is known about this topic?}

- The use of statins decreases the risk of venous thromboembolism (VTE).

- Studies on the association between lipid profile and VTE are inconsistent.

- Possibly inferior risk markers are used

\section{What does this paper add?}

- There is no association between either apolipoproteins or the classical lipoproteins and risk of VTE.

- The reported association between arterial cardiovascular disease and VTE appears not to be explained by an altered lipid profile as shared risk factor.

Hence, the use of anticoagulants was not included in our multivariable analyses. Third, the cohort under investigation is enriched for participants with higher levels of albuminuria. This enrichment might have reduced generalisability. However, it is unlikely to have influenced our risk estimates (i.e. HRs) on the relationship between (apo-) lipoproteins and VTE, as these estimates did not significantly change after accounting for study design. Generalisability might have also been slightly reduced due to the inclusion criteria of the PREVEND Study, in which inclusion was limited to subjects aged 28-75 years at baseline. This might explain the somewhat low mean age at time of VTE found in our study, when compared to other studies $(40,41)$. Fourth, lipid profile was assessed at inclusion. It is possible that lipid profile has changed during follow-up due to the natural course of lipid biomarkers or lifestyle intervention.

The present study is important in understanding the pathophysiology of VTE. Our results show no association between either apolipoproteins or the classical lipoproteins and VTE risk. Apparently, the reported association between arterial cardiovascular disease and VTE is not explained by an altered lipid profile as shared risk factor. Future studies should focus on other mechanisms that can explain the association between arterial cardiovascular disease and VTE to give us more insight into the pathophysiology of VTE.

\section{Acknowledgements}

The authors would like to thank Dr. G. Piersma-Wichers, head of the regional anticoagulation clinic, who provided us with essential data for this study.

\section{Conflicts of interest}

None declared.

\section{References}

1. Becattini C, Vedovati MC, Ageno W, et al. Incidence of arterial cardiovascular events after venous thromboembolism: a systematic review and a meta-analysis. J Thromb Haemost 2010; 8: 891-897.

2. Glynn RJ, Danielson E, Fonseca FA, et al. A randomized trial of rosuvastatin in the prevention of venous thromboembolism. N Engl J Med 2009; 360: 1851-1861.
3. Grady D, Wenger NK, Herrington D, et al. Postmenopausal hormone therapy increases risk for venous thromboembolic disease. The Heart and Estrogen/progestin Replacement Study. Ann Intern Med 2000; 132: 689-696.

4. Ray JG, Mamdani M, Tsuyuki RT, et al. Use of statins and the subsequent development of deep vein thrombosis. Arch Intern Med 2001; 161: 1405-1410.

5. Holst AG, Jensen G, Prescott E. Risk factors for venous thromboembolism: results from the Copenhagen City Heart Study. Circulation 2010; 121: 1896-1903.

6. Tsai AW, Cushman M, Rosamond WD, et al. Cardiovascular risk factors and venous thromboembolism incidence: the longitudinal investigation of thromboembolism etiology. Arch Intern Med 2002; 162: 1182-1189.

7. Everett BM, Glynn RJ, Buring JE, et al. Lipid biomarkers, hormone therapy and the risk of venous thromboembolism in women. J Thromb Haemost 2009; 7: 588-596.

8. Deguchi H, Pecheniuk NM, Elias DJ, et al. High-density lipoprotein deficiency and dyslipoproteinemia associated with venous thrombosis in men. Circulation 2005; 112: 893-899.

9. Doggen CJ, Smith NL, Lemaitre RN, et al. Serum lipid levels and the risk of venous thrombosis. Arterioscler Thromb Vasc Biol 2004; 24: 1970-1975.

10. von Depka M, Nowak-Gottl U, Eisert R, et al. Increased lipoprotein (a) levels as an independent risk factor for venous thromboembolism. Blood 2000; 96:3364-3368.

11. Parish S, Peto R, Palmer A, et al. The joint effects of apolipoprotein B, apolipoprotein A1, LDL cholesterol, and HDL cholesterol on risk: 3510 cases of acute myocardial infarction and 9805 controls. Eur Heart J 2009; 30: 2137-2146.

12. McQueen MJ, Hawken S, Wang X, et al. Lipids, lipoproteins, and apolipoproteins as risk markers of myocardial infarction in 52 countries (the INTERHEART study): a case-control study. Lancet 2008; 372: 224-233.

13. Emerging Risk Factors Collaboration, Di Angelantonio E, Sarwar N, et al. Major lipids, apolipoproteins, and risk of vascular disease. J Am Med Assoc 2009; 302: 1993-2000.

14. Hillege HL, Janssen WM, Bak AA, et al. Microalbuminuria is common, also in a nondiabetic, nonhypertensive population, and an independent indicator of cardiovascular risk factors and cardiovascular morbidity. J Intern Med 2001; 249 : 519-526.

15. Chobanian AV, Bakris GL, Black HR, et al. The Seventh Report of the Joint National Committee on Prevention, Detection, Evaluation, and Treatment of High Blood Pressure: the JNC 7 report. J Am Med Assoc 2003; 289: 2560-2572.

16. Expert Committee on the Diagnosis and Classification of Diabetes. Report of the Expert Committee on the Diagnosis and Classification of Diabetes Mellitus. Diabetes Care 1997; 20: 1183-1197.

17. Levey AS, Bosch JP, Lewis JB, et al. A more accurate method to estimate glomerular filtration rate from serum creatinine: a new prediction equation. Modification of Diet in Renal Disease Study Group. Ann Intern Med 1999; 130: 461-470.

18. Warnick GR, Nauck M, Rifai N. Evolution of methods for measurement of HDLcholesterol: from ultracentrifugation to homogeneous assays. Clin Chem 2001; 47: 1579-1596.

19. Friedewald WT, Levy RI, Fredrickson DS. Estimation of the concentration of lowdensity lipoprotein cholesterol in plasma, without use of the preparative ultracentrifuge. Clin Chem 1972; 18: 499-502.

20. Steinmetz J, Tarallo P, Fournier B, et al. Reference limits of apolipoprotein A-I and apolipoprotein B using an IFCC standardized immunonephelometric method. Eur J Clin Chem Clin Biochem 1995; 33: 337-342.

21. Survey Data Reference Manual Release 10. College Station, Texas: Stata Press; 2007.

22. Bova C, Marchiori A, Noto A, et al. Incidence of arterial cardiovascular events in patients with idiopathic venous thromboembolism. A retrospective cohort study. Thromb Haemost 2006; 96: 132-136.

23. Prandoni P, Bilora F, Marchiori A, et al. An association between atherosclerosis and venous thrombosis. N Engl J Med 2003; 348: 1435-1441.

24. Sorensen HT, Horvath-Puho E, Pedersen L, et al. Venous thromboembolism and subsequent hospitalisation due to acute arterial cardiovascular events: a 20-year cohort study. Lancet 2007; 370: 1773-1779.

25. Yu-Poth S, Zhao G, Etherton T, et al. Effects of the National Cholesterol Education Program's Step I and Step II dietary intervention programs on cardiovascular disease risk factors: a meta-analysis. Am J Clin Nutr 1999; 69: 632-646.

26. de Lorgeril M, Renaud S, Mamelle N, et al. Mediterranean alpha-linolenic acidrich diet in secondary prevention of coronary heart disease. Lancet 1994; 343 : 1454-1459.

27. Trichopoulou A, Costacou T, Bamia C, et al. Adherence to a Mediterranean diet and survival in a Greek population. N Engl J Med 2003; 348: 2599-2608. 
28. Hansen-Krone IJ, Enga KF, Njolstad I, et al. Heart healthy diet and risk of myocardial infarction and venous thromboembolism. The Tromsø Study. Thromb Haemost 2012; 108: 554-560.

29. Steffen LM, Folsom AR, Cushman M, et al. Greater fish, fruit, and vegetable intakes are related to lower incidence of venous thromboembolism: the Longitudinal Investigation of Thromboembolism Etiology. Circulation 2007; 115: 188-195.

30. Lutsey PL, Steffen LM, Virnig BA, et al. Diet and incident venous thromboembolism: the Iowa Women's Health Study. Am Heart J 2009; 157: 1081-1087.

31. Chamberlain AM, Folsom AR, Heckbert SR, et al. High-density lipoprotein cholesterol and venous thromboembolism in the Longitudinal Investigation of Thromboembolism Etiology (LITE). Blood 2008; 112: 2675-2680.

32. Walldius G, Jungner I. Is there a better marker of cardiovascular risk than LDL cholesterol? Apolipoproteins B and A-I--new risk factors and targets for therapy. Nutr Metab Cardiovasc Dis 2007; 17: 565-571.

33. Griffin JH, Fernandez JA, Deguchi H. Plasma lipoproteins, hemostasis and thrombosis. Thromb Haemost 2001; 86: 386-394.

34. Lacoste L, Lam JY, Hung J, et al. Hyperlipidemia and coronary disease. Correction of the increased thrombogenic potential with cholesterol reduction. Circulation 1995; 92: 3172-3177.
35. Ramcharan AS, Van Stralen KJ, Snoep JD, et al. HMG-CoA reductase inhibitors, other lipid-lowering medication, antiplatelet therapy, and the risk of venous thrombosis. J Thromb Haemost 2009; 7: 514-520.

36. Sen-Banerjee S, Mir S, Lin Z, et al. Kruppel-like factor 2 as a novel mediator of statin effects in endothelial cells. Circulation 2005; 112: 720-726.

37. Ridker PM, Rifai N, Clearfield M, et al. Measurement of C-reactive protein for the targeting of statin therapy in the primary prevention of acute coronary events. $\mathrm{N}$ Engl J Med 2001; 344: 1959-1965.

38. Ridker PM, Rifai N, Pfeffer MA, et al. Long-term effects of pravastatin on plasma concentration of C-reactive protein. The Cholesterol and Recurrent Events (CARE) Investigators. Circulation 1999; 100: 230-235.

39. Undas A, Brummel-Ziedins KE, Mann KG. Statins and blood coagulation. Arterioscler Thromb Vasc Biol 2005; 25: 287-294.

40. Naess IA, Christiansen SC, Romundstad P, et al. Incidence and mortality of venous thrombosis: a population-based study. J Thromb Haemost 2007; 5: 692-699.

41. Oger E. Incidence of venous thromboembolism: a community-based study in Western France. EPI-GETBP Study Group. Groupe d'Etude de la Thrombose de Bretagne Occidentale. Thromb Haemost 2000; 83: 657-660. 